\title{
Feather-Like Structures in Positive Streamers Interpreted as Electron Avalanches
}

\author{
Gideon Wormeester $^{1 *}$, Sander Nijdam ${ }^{2}$, and Ute Ebert ${ }^{1,2}$ \\ ${ }^{1}$ Centrum Wiskunde \& Informatica, PO Box 94079, 1090 GB, Amsterdam, The Netherlands \\ ${ }^{2}$ Department of Applied Physics, Eindhoven University of Technology, PO Box 513, 5600 MB, Eindhoven, The Netherlands
}

Received November 25, 2010; accepted March 28, 2011; published online August 22, 2011

In experiments positive streamers can have a feather-like structure, with small hairs connected to the main streamer channel. These feathers were observed in pure nitrogen (with impurities of $1 \mathrm{ppm}$ oxygen or less) but not in air. We hypothesize that these hairs are individual electron avalanches moving towards the streamer channel. Based on results of numerical simulations, we provide a theoretical explanation why these hairs are visible in nitrogen, but not in air. (C) 2011 The Japan Society of Applied Physics

\section{Introduction}

\subsection{Positive streamers}

Streamers are thin channels of ionized gas that are of significant importance in processes of atmospheric electricity (for example lightning and sprites ${ }^{1,2)}$ ) as well as in industrial applications such as lighting and disinfection. ${ }^{3)}$ We distinguish between positive and negative streamers. Negative streamers propagate in the direction of the electron drift, while positive streamers move against the drift direction and therefore require a source of electrons ahead of the streamer head. Yet positive streamers emerge more easily than negative streamers, which makes them more suitable for experiments.

The source of electrons in front of a positive streamer head is typically assumed to be photo-ionization, in particular in air, but this mechanism can be replaced by detachment from background ionization. In reality, both mechanisms are present. In recent work, ${ }^{4,5)}$ the dependence of the relative influence of each mechanism on parameters such as gas composition and repetition frequency of the discharges was investigated. Detachment from background ionization is only of influence in discharges with high repetition frequencies, in the $\mathrm{kHz}$ range for air and above $1 \mathrm{~Hz}$ in near-pure nitrogen. In all other cases, photo-ionization completely dominates the effect of any background ionization present in the gas.

\subsection{Effect of gas composition}

The amount of electrons generated by photo-ionization depends on the gas composition: In $\mathrm{N}_{2}: \mathrm{O}_{2}$-mixtures, the photo-ionization intensity depends both on the $\mathrm{N}_{2}$ density (for the number of emitted photons) and on the $\mathrm{O}_{2}$ density (that determines the absorption length). Therefore one expects streamers to be different in different $\mathrm{N}_{2}: \mathrm{O}_{2}$ mixtures. However, both in experiments ${ }^{4)}$ and in numerical simulations, ${ }^{5)}$ many of the streamer properties such as velocity and diameter are remarkably insensitive to order of magnitude changes in the number of photo-electrons outside the streamer.

Additionally, positive streamers have been observed in mixtures other than $\mathrm{N}_{2}: \mathrm{O}_{2}$, such as argon as well as mixtures representing the atmospheres of Jupiter and Venus, ${ }^{6}$ ) again with velocities that are very similar to the $\mathrm{N}_{2}: \mathrm{O}_{2}$ streamers. In these gases, the source of the electrons in front of the streamer head is not yet known. The lack of nitrogen and oxygen makes the classical $\mathrm{N}_{2}: \mathrm{O}_{2}$-photo-ionization mechan-

*E-mail address: G.Wormeester@cwi.nl

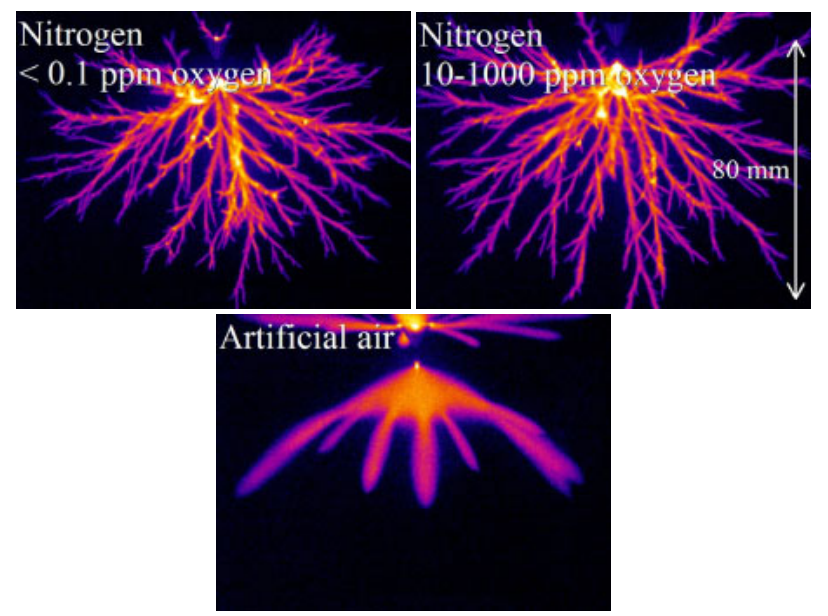

Fig. 1. (Color online) Streamers in three different $\mathrm{N}_{2}: \mathrm{O}_{2}$-mixtures at 200 mbar. The top-left panel shows a mixture with an oxygen fraction of at most $10^{-7}$, the top-right panel shows a less pure nitrogen source where the oxygen fraction is estimated to be between $10^{-3}$ and $10^{-5}$ and the bottom panel shows streamers in artificial air. In all cases, a positive voltage pulse of $25 \mathrm{kV}$ and of $130 \mathrm{~ns}$ duration is applied to the upper needle electrode (with the Blumlein pulser described in ref. 4), the distance to the plate electrode below is $16 \mathrm{~cm}$ and the upper $8 \mathrm{~cm}$ are shown. In the artificial air image, the brightness is four times more enhanced than in the nitrogen images.

ism in air impossible. Some form of photo-ionization from the gases in these atmospheres is plausible, but no detailed models are available yet.

While positive streamers in different gas-mixtures have many similar properties, there are clear differences. In "pure" nitrogen as well as in argon, Nijdam et al. ${ }^{4)}$ observed small hairs connecting to the main streamer channels, giving the entire channel a somewhat feather-like appearance. The nitrogen had at most $1 \mathrm{ppm}$ of oxygen contamination, while the argon had at most $10 \mathrm{ppm}$ of impurities. Independently, Takahashi et al. ${ }^{7)}$ observed the same phenomenon in streamers in argon. In air, no such feathers were observed. Figure 1 shows the difference in streamer smoothness and feather formation between air (bottom panel) and two nearpure nitrogen mixtures (top panels).

\section{Observations of Feather-Like Structures}

In experiments on streamers in $\mathrm{N}_{2}$ with at most $1 \mathrm{ppm} \mathrm{O}_{2}$ as well as in argon a feather-like structure was observed around the main streamer channels. ${ }^{4)}$ More recently, experiments have been done in even purer nitrogen, where the $\mathrm{O}_{2}$ contamination is at most $100 \mathrm{ppb}$. Lowering the oxygen 

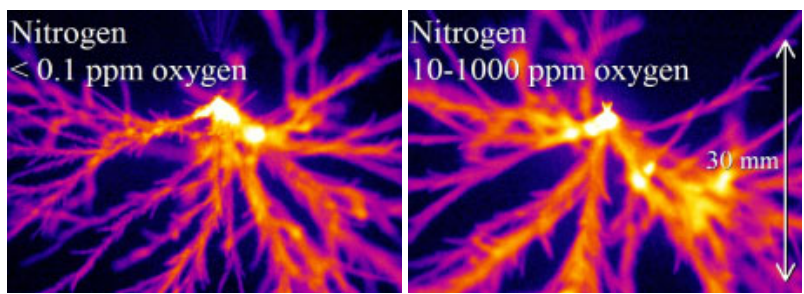

Fig. 2. (Color online) Comparison between feather-like structures in streamers in nitrogen with small oxygen admixtures. These images are closeups of the area near the needle for discharges under the same conditions as in Fig. 1. The left panel shows a mixture with an oxygen fraction of at most $10^{-7}$, the right panel shows a less pure nitrogen source where the oxygen fraction is estimated to be between $10^{-3}$ and $10^{-5}$.

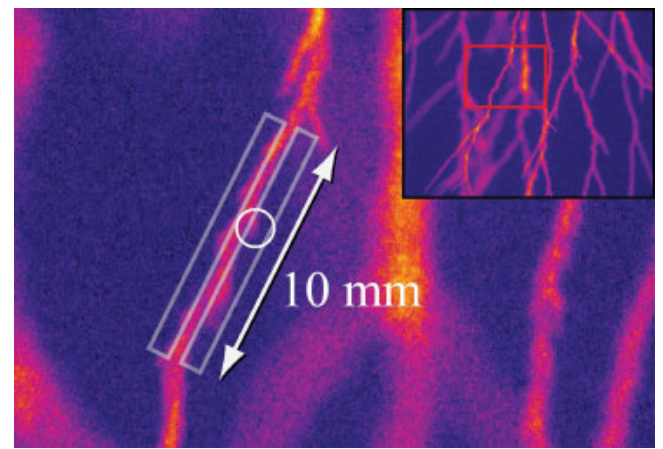

Fig. 3. (Color online) Zoom to segments of positive streamers in nitrogen with at most $1 \mathrm{ppm}$ of impurities at 200 mbar. The white circle contains an example of a hair. The rectangular boxes mark the area in which hairs are counted for the purpose of determining the number of hairs per $\mathrm{mm}$ of streamer length. The picture is from ref. 4

density decreases the smoothness of the streamer channel and makes the feather-like structure more apparant, as can be seen in Fig. 2, which consists of closeups of Fig. 1. The protrusions from the main streamer channel, like the one indicated by the circle in Fig. 2 will be called "hairs".

An estimate of the number of "hairs" in the feathers is made by drawing two boxes parallel to the streamer channel, as indicated in Fig. 3, and manually counting the number of visible hairs in these boxes. Dividing the hair-count by the length of the boxes yields the number of hairs per unit of streamer length. It must be noted that due to the two dimensional nature of the images, any hairs that are in front of or behind the streamer in the projection of the camera, will not be visible and will therefore not be counted. Consequently, the hair-count obtained by this method is a lower limit for the actual number of hairs.

Using this method, an estimate of about of 1 hair per mm of streamer length was found in the image plane of streamers in nitrogen with at most 1 ppm oxygen at 200 mbar. We remark that this is only an order-of-magnitude estimate due to the strong stochastic nature of the phenomenon and the diffculty of counting.

\section{Hypothesis on the Origin of Feathers}

\subsection{Hairs formed by avalanches}

We hypothesize that the feathers are separate electron avalanches moving towards the streamer. Free electrons in front of the positive streamer head drift towards the streamer due to the enhanced electric field generated by the space

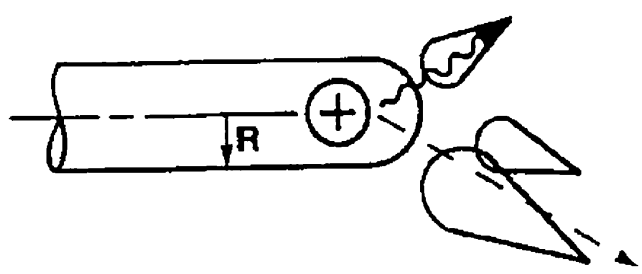

Fig. 4. Schematic representation of a positive streamer preceded by ionization avalanches as found in ref. 8 and many other papers and textbooks. The drawing can be traced back to Raether in $1939^{9)}$ and is discussed in more detail in ref. 10. We know now that the space charge is not uniformly distributed in the streamer head, but concentrated in a thin layer in contrast to the sketch. The number and role of the avalanches is discussed in this article.

charge layer in the streamer head. Once the electrons enter the region where the electric field is above the breakdown value (where the impact ionization rate exceeds the attachment rate), more free electrons are created than there are lost. This results in an avalanche that drifts towards the streamer head. This can be seen schematically in Fig. 4. If the avalanche is created directly in front of the streamer head, it will be overtaken by the propagating streamer and will therefore not be visible in experiments. Avalanches seen in experiments start away from the path of streamer propagation.

If at the critical electric field, where the impact ionization and attachment rates are equal, the electron density is low, one expects that some of these avalanches do not overlap and can be seen as distinct structures. On the other hand, if the electron density is already high enough so that the average distance between free electrons is much smaller than the dimensions of the streamer, one expects so many avalanches that they will overlap and can no longer be seen as separate entitites, but only as a continuum, being part of the propagating streamer.

\subsection{Effect of oxygen density on hair-formation}

The source of electrons in front and to the sides of the streamer head is assumed to be photo-ionization. An excited nitrogen molecule falls back to a state with lower energy, emitting a photon with the right energy to ionize an oxygen molecule. The characteristic ionization length of these photons scales inversely with the oxygen density and as a consequence significantly less photons will create ionization in the area around the streamer. Therefore the density of free electrons in the region away from the streamer head is expected to be orders of magnitude higher in air than in nitrogen with only a very small oxygen admixture. Therefore we expect avalanches to overlap more easily in air, while in nitrogen with a $1 \mathrm{ppm}$ oxygen admixture, the avalanches are more likely to be distinct; we will quantify this expectation in $\S 4$.

As the oxygen density decreases, so does the electron density outside the streamer channel. As a consequence, the avalanches that head towards the streamer channel become more and more rare, making stochastic effects visible, rather than forming a smooth channel. In experiments we see the channel being less smooth in the purest nitrogen with $<0.1 \mathrm{ppm}$ of oxygen (Fig. 2, left panel) than in nitrogen with an oxygen fraction between $10^{-3}$ and $10^{-5}$ (Fig. 2, right panel). 


\subsection{Distinction between hairs and branches}

At first glance, the formation of hairs connected to the streamer channel seems to be similar to the branching of streamer channels. However, in the model for hair-formation we propose, there are significant differences between hairs and branches. During a branching event, the streamer head is divided into fragments that due to electrostatic repulsion continue to propagate in different directions. Each fragment forms its own streamer channel with space charge layer, similar to the original streamer.

Hairs on the other hand do not form their own space charge layer. They consist of avalanches of electrons that are attracted by the positive charge in the streamer head. This property puts an upper bound on the length of the hairs, as they only start when the electric field exceeds the breakdown field. Streamer branches, on the other hand, generate their own enhanced electric field and can therefore propagate independently from the "parent" streamer. The branching of streamers is discussed in detail by Ebert et al. ${ }^{10,11)}$ They concluded that an instability of the space charge layer is a necessary ingredient of streamer branching.

Despite the differences between hairs and branches, the appearance and frequency of both phenomena is correlated. Mixtures that create a high electron density outside the streamer channel will see less branching and less hairs, due to the smoother structure of the streamer head. Liu and Pasko ${ }^{12)}$ as well as Luque et al. ${ }^{13)}$ found that photoionization suppresses branching. Experiments show that streamers branch more easily in near-pure nitrogen mixtures than they do in air. Similarly, streamers in argon branch more easily than streamers in air.

\section{Numerical Simulations and Results}

\subsection{Simulation model}

Numerical simulations were performed with a code using an adaptive grid refinement scheme. ${ }^{14)}$ The model consists of a fluid description for the densities with drift, diffusion and reaction terms. Included reactions are impact ionization, attachment, detachment and recombination. Photo-ionization is included using the widely used Zheleznyak model, ${ }^{15}$ ) implemented as described by Luque et al. ${ }^{13)}$ The electrode configuration is needle-plane. Simulations are done at standard temperature and pressure, using a voltage of $24 \mathrm{kV}$ with an $8 \mathrm{~mm}$ gap between the tip of the needle and the planar electrode. Initially, no charged species are present in the computational domain except for a Gaussian seed located at the needle tip, serving as the source of streamer inception. Further details of the model and its parameters are described in ref. 5.

Since we use a fluid approximation, our code can not be used to model individual avalanches. For this, a particle code or a hybrid code such as the one by Li et al. ${ }^{16)}$ is needed. However, we can still use results of the fluid code to draw conclusions on whether the presence of hairs and the corresponding feather-like structure can be expected or not.

\subsection{Effect of oxygen density}

The left panel of Fig. 5 shows the electron density and the boundary of the region where the electric field reaches the critical field and avalanches can occur; the simulation is in air at standard termperature and pressure. With the rate

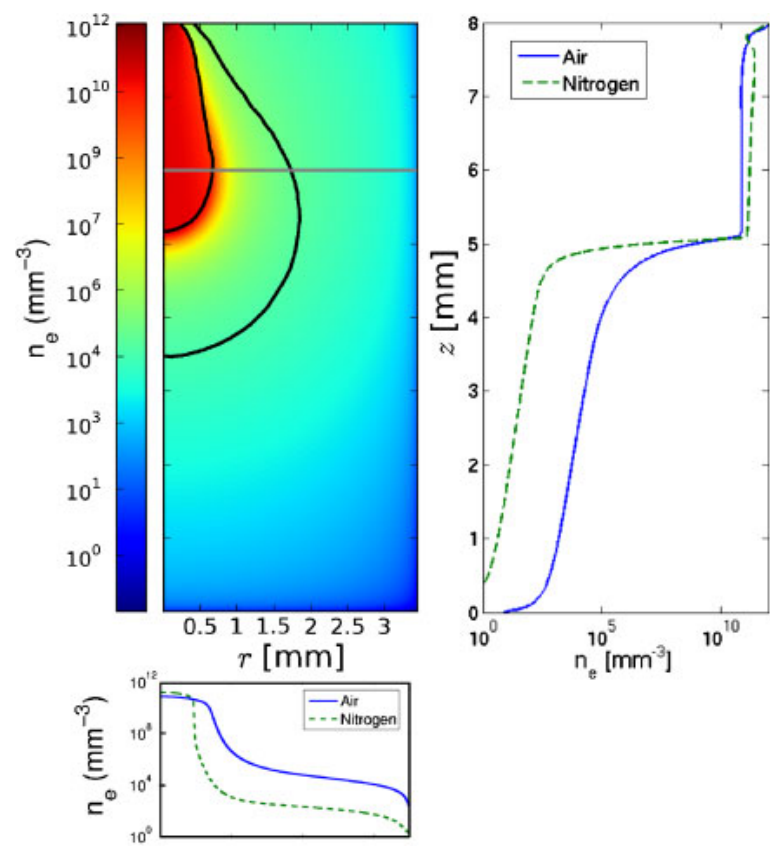

Fig. 5. (Color online) Left: Electron density in $\mathrm{mm}^{-3}$ of a positive streamer in air. The black lines indicate where the electric field reaches the breakdown value, so in the area between the black lines the field is above the breakdown value. Right: Electron density on the axis of positive streamers in air (solid line) and $\mathrm{N}_{2}$ with a 1 ppm $\mathrm{O}_{2}$ admixture (dashed line). Bottom: Electron density in $\mathrm{mm}^{-3}$ on a cross section of positive streamers in air (solid line) and $\mathrm{N}_{2}$ with a 1 ppm $\mathrm{O}_{2}$ admixture (dashed line). The gray line in the top-left panel indicates the location where the cross section was taken.

coefficients from BOLSIG $+{ }^{17)}$ for impact ionization and attachment, as they were used in the simulation, this critical field is $32 \mathrm{kV} \mathrm{cm}^{-1}$.

It is immediately obvious that at the outer boundary of the area where the electric field is above the breakdown field (see Fig. 5, left panel), the electron density is already $10^{5} \mathrm{~mm}^{-3}$. This will generate so many avalanches that they will overlap and are no longer distinct. Running the same simulation on a mixture of $\mathrm{N}_{2}$ with $1 \mathrm{ppm} \mathrm{O}_{2}$ gives completely different results. As can be seen in the right panel of Fig. 5, the electron density away from the streamer head is around $10^{2} \mathrm{~mm}^{-3}$. Additionally, the density of the attaching oxygen has been reduced by 5 orders of magnitude, so the critical field is lower than in air, around $20 \mathrm{kV} \mathrm{cm}^{-1}$ (rather than $32 \mathrm{kV} \mathrm{cm}^{-1}$ in air), which in turn means that avalanches can form further away from the streamer head. At these levels of electron-densities, $10^{2} \mathrm{~mm}^{-3}$, the number of avalanches in this nitrogen mixture will be low enough in number to be visually distinct, resulting in observable hairs in experiments.

In simulations performed at lower oxygen densities, the electron density away from the streamer head is even lower. With just $10 \mathrm{ppb}$ of $\mathrm{O}_{2}$, the electron density outside the streamer is almost 2 orders of magnitude lower than in the simulation with $1 \mathrm{ppm}$ of $\mathrm{O}_{2}$. At these levels of $\mathrm{O}_{2}$, the photo-ionization intensity is completely determined by the algebraic falloff of photon density when moving away from the photon source, the streamer head. With absorption lengths exceeding hundreds of meters, we can safely assume that the exponential falloff of photon density due to absorption events does not play a role. 
Since we expect that the electron density outside the streamer channel is predominantly determined by the photoionization intensity, we expect that in these low levels of oxygen, the electron density outside the streamer is proportional to the oxygen density. The fact that this proportionality does not hold up completely is due to electron losses from attachment to oxygen. In mixtures with more oxygen, more of the electrons generated by photo-ionization are attached and consequently, the electron density is lower than the simple proportionality argument would predict.

We must remark that at oxygen densities as low as $10 \mathrm{ppb}$ (and corresponding to low electron densities outside the streamer), the density approximation used in our fluid model is no longer valid outside the streamer as the electron density in each cell becomes too low and stochastic effects start to play a role. Also, in our simulations and estimates we have ignored the effects of background ionization present in the domain. However, for these low electron densities, detachment from background ionization may be a non-negligible contribution to the electron density outside the streamer channel.

\subsection{Estimating the number of hairs}

We can estimate the number of hairs per mm of streamer length for our simulation in nitrogen with $1 \mathrm{ppm}$ of oxygen by counting the number of free electrons outside the streamer. From our simulations, we can obtain the electron density in each cell of the simulation domain. In the simulation with $1 \mathrm{ppm}$ of $\mathrm{O}_{2}$, we have an electron density of the order of $10^{2} \mathrm{~mm}^{-3}$ to the sides of the streamer. However, the oxygen level in the experiments was probably about $0.2 \mathrm{ppm}$ (with a total impurity below $1 \mathrm{ppm}$ ), whereas the simulations used $1 \mathrm{ppm}$. At low oxygen levels, the electron density outside the streamer is roughly proportional to the oxygen density. ${ }^{5)}$ So we would have approximately 20 electrons per $\mathrm{mm}^{-3}$ to the sides of the streamer in the conditions of the experiment. This translates to a distance between electrons of approximately $0.3 \mathrm{~mm}$. We therefore expect to see approximately 3 hairs per $\mathrm{mm}$ of streamer length.

In experiments in nitrogen at $200 \mathrm{mbar}$, about 1 hair per $\mathrm{mm}$ of streamer length was observed in the image plane. According to scaling laws for streamers, ${ }^{2)}$ the number of hairs does not depend on the pressure. This is because electron densities scale as the square of the gas density, while similar lenghts scale as the inverse of the gas density.

Our theoretical estimate is only a rough estimate for the following reasons: Since our numerical code assumes cylindrical symmetry, a streamer can only be followed up to the point where it branches. This limits the length of streamers in nitrogen that we can simulate and therefore we can't directly match the parameters, such as electrode-gap, used in experiments. Furthermore, the individual hairs can't be simulated with a fluid code, which greatly limits the extent to which we can compare our estimate with experimental results.

\section{Conclusions}

Even though many streamer properties are remarkably insensitive to changes in gas composition, the formation of feather-like structures, small hairs connected to the main streamer channel, appears to be sensitive to the background gas. In air, such hairs were not observed, while in $\mathrm{N}_{2}: \mathrm{O}_{2}$ mixtures with very small (less than $10^{-3}$ ) $\mathrm{O}_{2}$ fraction, the feather-like structures are visible. As the oxygen density is decreased, the feather-like structure becomes more pronounced as the streamer channels become less smooth.

We have proposed that the feather-like structures in positive streamers are due to individual avalanches created by photo-ionization. As a photo-electron moves into the region where the electric field exceeds the breakdown field, repeated impact ionization creates an electron avalanche that drifts towards the positive charge in the streamer head. Since in air, the absorption length for photo-ionization is much smaller than in near-pure nitrogen, the electron density outside the streamer head is much higher and consequently avalanches overlap and are not visible as distinct hairs. In near-pure nitrogen, the electron densities outside the streamer head are much lower and individual avalanches may be seen. The numerical simulations with a fluid model, while not suited for simulation of individual hairs, have provided evidence that supports our explanation of the effect of the $\mathrm{N}_{2}: \mathrm{O}_{2}$-ratio on the formation of hairs and feathers in positive streamers.

Furthermore, we have provided an estimate for the number of hairs in nitrogen with $1 \mathrm{ppm}$ of oxygen and compared it with the experimental value. Our estimate has the same order of magnitude as the experimental result, but limitations of the simulation model limit the accuracy of this estimate. A particle model is required to study the phenomenon of feather-formation in more detail.

\section{Acknowledgment}

G.W. acknowledges support by STW-project 10118, part of the Netherlands' Organization for Scientific Research (NWO).

1) V. P. Pasko: Plasma Sources Sci. Technol. 16 (2007) S13.

2) U. Ebert, S. Nijdam, C. Li, A. Luque, T. M. P. Briels, and E. M. van Veldhuizen: J. Geophys. Res. 115 (2010) A00E43.

3) E. J. M. van Heesch, G. J. J. Winands, and A. J. M. Pemen: J. Phys. D 41 (2008) 234015

4) S. Nijdam, F. M. J. H. van de Wetering, R. Blanc, E. M. van Veldhuizen, and U. Ebert: J. Phys. D 43 (2010) 145204.

5) G. Wormeester, S. Pancheshnyi, A. Luque, S. Nijdam, and U. Ebert: J. Phys. D 43 (2010) 505201.

6) D. Dubrovin, S. Nijdam, E. M. van Veldhuizen, U. Ebert, Y. Yair, and C. Price: J. Geophys. Res.: Space Phys. 115 (2010) A00E34.

7) E. Takahashi, S. Kato, A. Sasaki, Y. Kishimoto, and H. Furutani: J. Phys. D 44 (2011) 075204.

8) L. Niemeyer, L. Ullrich, and N. Wiegart: IEEE Trans. Electr. Insul. 24 (1989) 309.

9) H. Raether: Z. Phys. 112 (1939) 464 [in German].

10) U. Ebert, C. Montijn, T. M. P. Briels, W. Hundsdorfer, B. Meulenbroek, A Rocco, and E. M. van Veldhuizen: Plasma Sources Sci. Technol. 15 (2006) S118.

11) U. Ebert, F. Brau, G. Derks, W. Hundsdorfer, C.-Y. Kao, C. Li, A. Luque B. Meulenbroek, S. Nijdam, V. Ratushnaya, L. Schäfer, and S. Tanveer: Nonlinearity 24 (2011) C1.

12) N. Liu and V. P. Pasko: J. Geophys. Res. 109 (2004) A04301.

13) A. Luque, U. Ebert, C. Montijn, and W. Hundsdorfer: Appl. Phys. Lett. 90 (2007) 081501.

14) C. Montijn, W. Hundsdorfer, and U. Ebert: J. Comput. Phys. 219 (2006) 801.

15) M. Zheleznyak, A. Mnatsakanyan, and S. Sizykh: High Temp. 20 (1982) 357.

16) C. Li, U. Ebert, and W. Hundsdorfer: J. Phys. D 42 (2009) 202003.

17) G. J. M. Hagelaar and L. C. Pitchford: Plasma Sources Sci. Technol. 14 (2005) 722. 